\title{
IMPACT OF LEFT VENTRICULAR VOLUME OVERLOAD AND SYSTOLIC PULMONARY ARTERY PRESSURE ON RIGHT VENTRICULAR SYSTOLIC FUNCTION IN PATIENTS WITH CHRONIC PRIMARY MITRAL AND AORTIC REGURGITATION USING THREE DIMENSIONAL ECHOCARDIOGRAPHY
}

\author{
Hoda Shehata, Sanaa Ashour, Mohamed Ayman Abdelhay) \\ Department of Cardiology, Faculty of Medicine, Alexandria University, Alexandria, Egypt
}

\section{ABSTRACT}

Background. To compare the effects of left ventricular volume(LV) overload due to chronic organic mitral(MR) or aortic valve(AR) regurgitation on RV shape and function.

Methods: We studied 40 patients with moderate-severe or severe primary MR ( $60 \pm 19$ years, $52 \%$ were men) and 20 patients with moderate or severe AR (53 \pm 18 years, $81 \%$ were men). Three-dimensional echocardiography of LV and RV was performed to measure volumes and EF. RV fractional area change (FAC) was calculated and RV shape was assessed by calculating RV eccentricity index .

Results: LV end-diastolic volume (EDVi) was significantly larger in the patients with AR than those with MR. RV EF and RV FAC were lower in pts with AR than in those with MR. RV eccentricity index was significantly higher in the AR group ( $2.5 \pm 0.6$ vs $2.1 \pm 0.5, p=0.003)$. In both groups, LV EDVi showed positive correlations with RV shape (RV eccentricity: $r=0.693$ for $A R$ and $r=0.399$ for MR; $p<0.001$ ) and negative correlations with RV function (RV EF: $r=-0.545$ for $A R$ and $r=-0.383$ for MR, $p<0.001$; RV FAC: $r=-0.816$ for $\mathrm{AR}$ and $\mathrm{r}=-0.647$ for $\mathrm{MR}$, $\mathrm{p}<0.001)$.Moreover, LV sphericity index showed negative correlations with RV function (RVFAC: $r=-0.512$ for $M R$ and $r=-0.608$ for AR,P=0.001;RV EF:r=-0.408 for MR and $r=-0.469, P=0.004)$ and positive correlation with the RV shape (RV eccentricity index, $\mathrm{r}=0.39$ for MR and $\mathrm{r}=0.511, \mathrm{P}<0.001)$. On multivariable linear regression analysis, LV EDVi and LV sphericity index were found to be the only independent predictors of RV eccentricity index, EF and FAC.

Conclusions: RV remodeling in chronic LV overload due to MR or AR occurs independent on PASP values. LV size and shape are the only independent predictors of RV geometry and function. Accordingly, chronic AR has a greater impact on RV than MR.

Keywords: Left ventricle- Mitral regurgitation- Aortic regurgitation-Right ventricle.

\section{INTRODUCTION}

Ventricular function is an important predictor of preoperative and postoperative outcome of mitral and aortic valve regurgitation. This is well known in regard to left ventricular
(LV) function, which figures prominently in the clinical guidelines for surgical indications, ${ }^{(1,2)}$ but it is less well known in regard to right ventricular (RV) function. The RV performance may be influenced by elevated systolic pulmonary artery pressure (PASP), which is a common finding in degenerative severe chronic mitral regurgitation (MR).$^{(3-5)}$ On the other side, RV systolic dysfunction can be an indirect consequence of chronic LV volume overload and, thus of enlarged LV in MR patients. ${ }^{(6)}$ However, Elevation of PASP secondary to isolated aortic valve regurgitation (AR) is less common than with other valve lesions. ${ }^{(7)}$ Therefore, the aim of the present study is to compare effects of LV volume overload due to chronic organic MR or AR on RV shape and function and to assess the pathophysiologic determinants of RV systolic function .

\section{METHODS}

Study population:

Forty patients with moderate-severe or severe organic mitral valve regurgitation and twenty patients with moderate or severe aortic valve regurgitation were included in this study.

Standard transthoracic echocardiography was performed using a Vivid E9 and Vivid E 95 ultrasound system, (GE Vingmed Ultrasound AS, Horten, Norway) equipped with a M5S 3.5$\mathrm{mHz}$ transducer.

RV function was estimated by:1) tricuspid annular plane systolic excursion (TAPSE), 2)fractional area change (FAC). 
An apical 3D dataset of LV and RV was obtained using a Vivid E9 or Vivid E95 equipped with a 4V probe.(GE Vingmed, Horten, Norway).

LV and RV full-volume datasets were obtained from 4 or 6 consecutive beats for adequate temporal resolution (2040 volumes/sec).

Quantification of 3D LV volumes and EF was performed using a dedicated software (4D AutoLVQ, GE Vingmed, Norway). LV sphericity index is a quantitative parameter reflecting LV shape, calculated as the ratio between the LV end-diastolic volume divided by the volume of a sphere, whose diameter is equal to the longitudinal LV axis measured at end-diastole.

RV volumes and EF were measured using software packages designed for volumetric analysis (TomTec Imaging Systems GmbH, Unterschleissheim, Germany). RV shape was assessed using RV eccentricity index , calculated as the ratio between RV longest lateral distance to the perpendicular distance from RV septum to free wall - at the level of LV papillary muscles.

\section{STATISTICAL ANALYSIS}

Data were expressed as mean \pm standard deviation for continuous variables and as percentages for categorical variables. Between-group comparisons were performed using Student's t-tests or $\mathrm{x} 2$ test, as appropriate. To identify pathophysiological correlates of RV function and shape, univariate linear regression analysis was carried out. Candidate variables were entered into a stepwise multivariate regression analysis to identify the independent predictors of RV changes. Statistical significance was defined as $p<0.05$. Statistical analysis was performed using SPSS Version 20.0

\section{RESULTS:}

Baseline clinical and echocardiographic characteristics of the patient population are shown in (table 1, figure 1,2). Both LV end-diastolic volume (EDVi) and end-systolic volume (ESVi) were significantly larger in the patients with AR than those with MR. LV EF was significantly lower in AR group than in MR group.(table 2,3).

Table 1: Basic clinical and echocardiographic characteristics in MR and AR patients:

\begin{tabular}{lccc}
\hline & $\begin{array}{c}\text { MR group } \\
(\mathbf{n = 4 0})\end{array}$ & $\begin{array}{c}\text { AR group } \\
(\mathbf{n = 2 0})\end{array}$ & P value \\
\hline Male (\%) & 52 & 81 & 0.004 \\
\hline Age (y) & $60 \pm 14$ & $53 \pm 18$ & 0.008 \\
Min-Max & $20-89$ & $19-83$ & 0.1 \\
Mean \pm SD & $1.8 \pm 0.2$ & $1.9 \pm 0.2$ & 0.326 \\
\hline BSA (m) & $71 \pm 13$ & $71 \pm 11$ & $<0.001^{*}$ \\
\hline HR (Beats/min) & $126 \pm 16$ & $134 \pm 20$ & $<0.001^{*}$ \\
\hline Blood pressure & $75 \pm 7$ & $62 \pm 8$ & \\
Systolic (mmHg) & 8 & 30 & 15 \\
\hline Diastolic(mmHg) $(\mathbf{m})$ & & \\
\hline Atrial fibrillation(\%) & 75 & 22 & \\
\hline NYHA II(\%) & & & \\
\hline NYHA III-IV $(\%)$ & & & \\
\hline
\end{tabular}

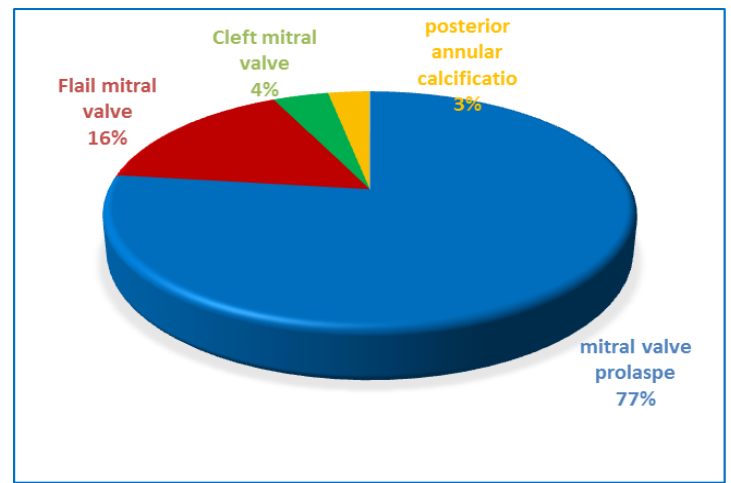

Figure (1): Etiology of MR

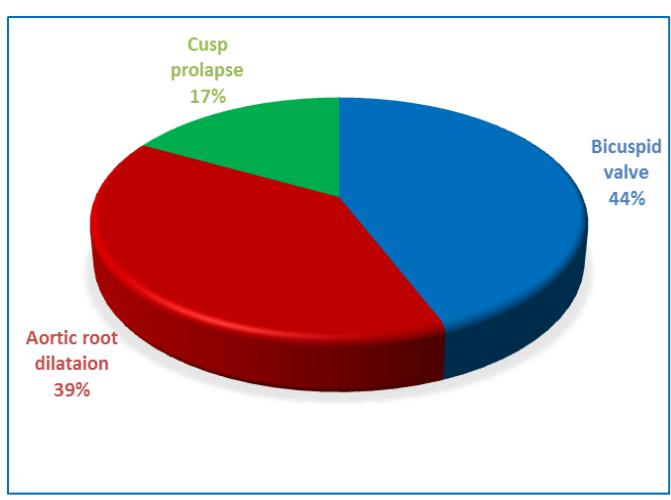

Figure (2) Etiology of AR 
Table 2:3D LV volumes, EF and sphericity index in MR and AR patients:

\begin{tabular}{lccc}
\hline LV geometry and function & MR $(\mathbf{n = 4 0})$ & AR(n=20) & P value \\
\hline 3D LVEDV $(\mathbf{m l})$ & $137 \pm 38$ & $205 \pm 84$ & $<0.001$ \\
\hline 3D LVESV $(\mathbf{m l})$ & $50 \pm 17$ & $97 \pm 59$ & $<0.001$ \\
\hline 3D LVEDVi $(\mathbf{m l} / \mathbf{m})$ & $78 \pm 20$ & $106 \pm 36$ & $<0.001$ \\
\hline 3D LVESVi $(\mathbf{m l} / \mathbf{m})$ & $29 \pm 8$ & $50 \pm 28$ & $<0.001$ \\
\hline LV EF $(\%)$ & $62 \pm 6$ & $54 \pm 10$ & $<0.001$ \\
\hline LV sphericity index & $0.49 \pm 0.10$ & $0.53 \pm 0.11$ & $<0.001$ \\
\hline
\end{tabular}

Table 3: RV volumes, function and eccentricity index in MR and AR patients:

\begin{tabular}{lccc}
\hline RV geometry and function & MR $(\mathbf{n = 4 0})$ & AR(n=20) & P value \\
\hline TAPSE $(\mathbf{m m})$ & $18 \pm 4$ & $19 \pm 4$ & 0.09 \\
\hline RV EDVi $(\mathbf{m l} / \mathbf{m} \mathbf{)}$ & $51 \pm 13$ & $59 \pm 12$ & $<0.001$ \\
\hline RV ESVi $(\mathbf{m l} / \mathbf{m})$ & $28 \pm 9$ & $31 \pm 9$ & 0.245 \\
\hline RV FAC $(\boldsymbol{\%})$ & $37 \pm 9$ & $34 \pm 9$ & 0.04 \\
\hline RV EF $(\%)$ & $43 \pm 7$ & $40 \pm 6$ & 0.032 \\
\hline RV eccentricity index & $2.1 \pm 0.5$ & $2.5 \pm 0.6$ & 0.003 \\
\hline
\end{tabular}

Pathophysiologic correlates of RV geometry and function: (Table 4)

In univariate analysis, LV EDVi showed significant positive correlations with RV eccentricity index $(r=0.693$ for $\mathrm{AR}$ and $\mathrm{r}=0.399$ for $\mathrm{MR}$; $\mathrm{p}<0.001)$ and negative correlations with RV EF (RV EF: $r=-0545$ for AR and $\mathrm{r}=-0.383$ for MR, $\mathrm{p}<0.001)$ and RV FAC (RV FAC: $\mathrm{r}=-$ 0.816 for $A R$ and $r=-0.647$ for $M R, p<0.001)$.

Moreover, LV sphericity index showed negative correlations with RV FAC $(r=-0.512$ for $\mathrm{MR}$ and $\mathrm{r}=-$ 0.608 for $\mathrm{AR}, \mathrm{P}=0.001)$ and with $\mathrm{RV} \mathrm{EF}(\mathrm{r}=-0.408$ for $\mathrm{MR}$ and $\mathrm{r}=-0.469$ for $\mathrm{AR}, \mathrm{P}=0.004)$ and positive correlation with the RV eccentricity index $(r=0.39$ for $M R$ and $\mathrm{r}=0.511$ for $\mathrm{AR}, \mathrm{P}<0.001$ ).

Regarding correlation with strain in both groups, in the AR group, LV GLS and peak RV LS showed significant positive correlation with RV EF but the MR group, only peak RV LS and mid septal RV LS showed significant positive correlation with RV EF.

Predictors of RV remodelling in LV overload: (Table 5) On multivariable linear regression analysis, LV EDVi and LV sphericity index were found to be the only independent predictors of RV eccentricity index, EF and FAC. However, RV EF, FAC and eccentricity index changes occur independent on PASP values.

Table 4:Correlation between different parameters and RV EF, RV FAC and eccentricity index

\begin{tabular}{|c|c|c|c|c|c|c|}
\hline & \multicolumn{6}{|c|}{$\mathbf{R V}$} \\
\hline & \multicolumn{2}{|c|}{$\mathbf{E F}$} & \multicolumn{2}{|c|}{ FAC } & \multicolumn{2}{|c|}{ Eccentricity index } \\
\hline & $\mathbf{r}$ & $\mathbf{P}$ & $\mathbf{r}$ & $\mathbf{P}$ & $\mathbf{r}$ & $\mathbf{p}$ \\
\hline \multicolumn{7}{|l|}{ MR group } \\
\hline 3D LV EDVi $\left(\mathrm{ml} / \mathrm{m}^{2}\right)$ & $-0.393^{*}$ & $0.001^{*}$ & $-0.759^{*}$ & $<0.001^{*}$ & $0.591^{*}$ & $<0.001^{*}$ \\
\hline LV Sphericity index & $-0.438^{*}$ & $<0.001^{*}$ & $-0.531^{*}$ & $<0.001^{*}$ & $0.441^{*}$ & $<0.001^{*}$ \\
\hline Basal septal LS (\%) & $0.356^{*}$ & $0.004^{*}$ & 0.004 & 0.974 & 0.131 & 0.306 \\
\hline Mid septal LS (\%) & $0.354^{*}$ & $0.004^{*}$ & 0.015 & 0.9070 & 0.200 & 0.116 \\
\hline LV GLS (\%) & 0.074 & 0.565 & 0.002 & 0.987 & -0.082 & 0.521 \\
\hline Peak RV GLS (\%) & $0.484^{*}$ & $<0.001^{*}$ & $0.248^{*}$ & $0.050^{*}$ & 0.171 & 0.181 \\
\hline \multicolumn{7}{|l|}{ AR group } \\
\hline 3D LV EDVi $\left(\mathrm{ml} / \mathrm{m}^{2}\right)$ & $-0.545-$ & $0.001^{*}$ & $-0.816^{*}$ & $<0.001^{*}$ & $0.693^{*}$ & $<0.001^{*}$ \\
\hline LV Sphericity index & $-0.469^{*}$ & $0.004^{*}$ & $-0.608^{*}$ & $<0.001^{*}$ & $0.511^{*}$ & $0.001^{*}$ \\
\hline Basal septal LS (\%) & 0.230 & 0.178 & 0.230 & 0.178 & 0.104 & 0.546 \\
\hline Mid septal LS (\%) & 0.164 & 0.338 & 0.072 & 0.678 & 0.069 & 0.689 \\
\hline LV GLS (\%) & $0.526^{*}$ & $0.001^{*}$ & $0.475^{*}$ & $0.003^{*}$ & $0.489^{*}$ & $0.002^{*}$ \\
\hline Peak RV GLS (\%) & $0.511^{*}$ & $0.001^{*}$ & $0.389^{*}$ & $0.019^{*}$ & $0.416^{*}$ & $0.012^{*}$ \\
\hline
\end{tabular}

r: Pearson coefficient

*: Statistically significant at $\mathrm{p} \leq 0.05$ 
Table 5: Echocardiographic predictors of the RV FAC, RV EF and RV eccentricity index by multivariate analysis

\begin{tabular}{|c|c|c|c|c|c|}
\hline \multirow{2}{*}{ RV FAC (\%) } & \multirow{2}{*}{ B } & \multirow{2}{*}{$\mathbf{t}$} & \multirow{2}{*}{ Sig. } & \multicolumn{2}{|c|}{$95 \%$ C.I } \\
\hline & & & & Lower & Upper \\
\hline 3D LV EDVi (ml/m2) & -0.181 & $7.052^{*}$ & $<0.001^{*}$ & -0.232 & -0.130 \\
\hline sPAP (mmHg) & -0.096 & $2.032^{*}$ & $0.045^{*}$ & -0.189 & -0.002 \\
\hline LV sphericity index & -18.525 & $2.527^{*}$ & $0.013^{*}$ & -33.081 & -3.969 \\
\hline $\operatorname{LV}$ EF $(\%)$ & -0.059 & 0.746 & 0.457 & -0.217 & 0.099 \\
\hline \multicolumn{6}{|l|}{$\mathrm{F}=31.395^{*} \mathrm{p}<0.001^{*} \mathrm{R}^{2}=0.572$} \\
\hline \multirow{2}{*}{ RV EF (\%) } & \multirow{2}{*}{ B } & \multirow{2}{*}{$\mathbf{t}$} & \multirow{2}{*}{ Sig. } & \multicolumn{2}{|c|}{ 95\% C.I } \\
\hline & & & & Lower & Upper \\
\hline 3D LV EDVi (ml/m2) & -0.074 & $2.782^{*}$ & $0.007^{*}$ & -0.126 & -0.021 \\
\hline sPAP (mmHg) & -0.089 & 1.829 & 0.071 & -0.185 & 0.008 \\
\hline LV sphericity index & -15.285 & $2.022^{*}$ & $0.046^{*}$ & -30.295 & -0.274 \\
\hline LV EF (\%) & 0.101 & 1.236 & 0.219 & -0.061 & 0.264 \\
\hline \multicolumn{6}{|l|}{$\mathrm{F}=10.340^{*} \mathrm{p}<0.001^{*} \mathrm{R}^{2}=0.306$} \\
\hline \multirow{2}{*}{ RV eccentricity index } & \multirow{2}{*}{ B } & \multirow{2}{*}{$\mathbf{t}$} & \multirow{2}{*}{ Sig. } & \multicolumn{2}{|c|}{ 95\% C.I } \\
\hline & & & & Lower & Upper \\
\hline 3D LV EDVi (ml/m2) & 0.011 & 6.530 & $<0.001^{*}$ & 0.008 & 0.015 \\
\hline sPAP (mmHg) & 0.005 & 1.692 & 0.094 & -0.001 & 0.012 \\
\hline LV sphericity index & 0.465 & 0.937 & $0.035^{*}$ & -0.520 & 1.451 \\
\hline LV EF (\%) & -0.002 & -0.434 & 0.665 & -0.013 & 0.008 \\
\hline $\mathrm{F}=22.934^{*} \mathrm{p}<0.001^{*} \mathrm{R}^{2}=0.494$ & & & & & \\
\hline
\end{tabular}

F: F test (ANOVA)

$\mathrm{R}$ : coefficient or regression

B: Unstandardized Coefficients

t: t-test of significance

CI: Confidence interval

LL: Lower limit

UL: Upper Limit

$*$ : Statistically significant at $\mathrm{p} \leq 0.05$

\section{DISCUSSION}

LV size and shape affect RV function and geometry .First ,the LV acts on RV function through the interventricular septum. ${ }^{(8)}$ Septal contraction contributes to both RV and $\mathrm{LV}$ functions and is regarded as one of the major determinants of RV performance. ${ }^{(9)}$ Second, both ventricles are enclosed inside relatively inextensible pericardium .Chronic LV volume overload owing to MR and AR results in eccentric hypertrophy with geometric changes of the LV .Thus, LV enlargement into more spherical shape impinges the RV with impairment of the septal function. Moreover, LV enlargement can cause septal shift to the right and this decreases the septo-free wall diameter of the RV and subsequently, increases RV eccentricity index.

Third, RV systolic function may be influenced by PASP elevation caused by primary or secondary pulmonary diseases and by left heart failure. ${ }^{(10)}$ Elevated LV filling pressure and left atrial pressure induces a backward rise in pulmonary capillary wedge pressure and artery pressure .In addition to the direct effect, pulmonary vascular remodeling or abnormal vasoconstriction contributes to PASP elevation. ${ }^{(11)}$

RV function and pulmonary artery systolic pressure:

The degree to which afterload, primarily a function of pulmonary artery pressure, affects RV EF is a controversial topic. In chronic organic MR, many studies have showed that RV performance is influenced by PASP with an inverse relation of RV EF to the level of PASP. ${ }^{(12,13)}$ Le Tourneau et al, found an independent relation of PASP to RV EF but this relation was weak, and this clearly suggests that PASP is not the main determinant of RV function in chronic organic MR. ${ }^{(6)}$ Moreover, elevated PASP $>45 \mathrm{mmHg}$ was detected in $30-40 \%$ of the patients referred for MV surgery. ${ }^{(4-6)}$

In chronic $\mathrm{AR}$, a recent study has shown that PASP elevation $>45 \mathrm{mmHg}$ was detected only in $14 \%$ of the patients who were indicated for aortic valve replacement and it was found that pulmonary hypertension was associated with high surgical risk and poor survival at three year follow up. ${ }^{(14)}$ Another recent study studied the long term outcomes in patients with $\mathrm{AR}$, mean value of 
their RV systolic pressure was $32 \pm 10 \mathrm{mmHg}$ and $\mathrm{RV}$ systolic dysfunction was detected in only $1.5 \%$ of the patients .However, the mean value of $\mathrm{LV}$ end diastolic diameter was $54 \pm 9 \mathrm{~mm}$ with only $11 \%$ of the patients who had severe dilatation $>65 \mathrm{~mm}$. $^{(15)}$

Although our study revealed that RV systolic dysfunction (RV EF $<45 \%$ and FAC $<35 \%$ ) was detected in $63 \%$ of patients with AR and in $58 \%$ of patients with MR, PASP elevation $>45 \mathrm{mmHg}$ was seen only in $8 \%$ of the former and $27 \%$ of the latter.

\section{RV function and left ventricular remodeling :}

LV sphericity is one of the echocardiographic indices that have been used to assess the changes in LV geometry.In chronic MR due to mitral valve prolapse, ischemic heart disease and rheumatic heart diseases that underwent mitral valve replacement, it was found that LV geometric changes assessed by LV sphericity index are independent risk factors for survival. ${ }^{(16)}$ Moreover, In dogs with congestive heart failure related to severe MR, LV enlargement compresses and flattens the RV, thereby, impairing RV function . ${ }^{(17)}$ In AR, an experimental animal study concluded that LV sphericity index predicts LV systolic dysfunction in aortic regurgitation..$^{(18)}$

There is an important concept of ventricular interdependence between the RV and LV. Thus, the changes in pressure or volume in one chamber are likely to affect the pressure and the size of the other. ${ }^{(19)}$ The explanations of this are the sharing of both ventricles by the interventricular septum (IVS), the insertion of anterior and posterior ends of the RV free wall into the IVS and encircling fibers of the pericardium .So, in chronic volume overload due to MR or AR , the LV enlarges and changes from ellipsoid into more spherical shape, thus impinges the RV and impair the septal contraction. In our study, we found that both LV EDVi and LV sphericity index are increased in both MR and AR but they were significantly larger in the AR group. So upon correlation between both LV EDVi and LV sphericity index and RV systolic function assessed by RV EF and FAC, there was significant negative correlations between them in both groups with stronger correlations in patients with AR when compared to those with MR. Accordingly, AR has a greater impact on the RV function.

\section{$R V$ shape and $L V$ remodeling:}

$\mathrm{RV}$ eccentricity index is a novel echocardiographic index that is designed to assess the RV shape changes. It shares the same geometric concept with LV eccentricity index that have been used in several studies to differentiate between RV pressure and volume overload. ${ }^{(20,21)}$

Our study revealed that RV eccentricity was significantly higher in the AR group when compared to the MR group .Upon correlation with LV EDVi and LV sphericity index , both have a significant positive correlation with RV eccentricity index, and this correlation was stronger in the AR group.
Interestingly, we have found a strong negative correlation between RV eccentricity index and both RV EF and FAC. $(\mathrm{r}=-0.45$ for RV EF and -0.52 for $\mathrm{FAC}, \mathrm{p}<0.001)$

According to these results, AR group had more RV systolic impairment and more eccentric RV than MR group.

\section{CONCLUSION:}

RV systolic dysfunction with EF less than $45 \%$ is a common finding in patients with chronic moderate or severe MR and AR and this can be partially explained by PASP elevation. However, RV function can be influenced by other interactions as demonstrated by the association of $\mathrm{LV}$ enlargement and $\mathrm{RV} \mathrm{EF}$ and FAC .In relatively inextensible pericardium, LV enlargement into more spherical shape impinges the RV with impairment of the septal function. Moreover, LV enlargement can cause septal shift to the right and this reduces the distance from the RV septum to the RV free wall, causes more elongation in the antero-posterior diameter and thus increases the RV eccentricity index.

RV remodeling in chronic $L V$ overload due to MR or AR occurs independent on PASP values. LV size and shape are the only independent predictors of RV geometry and function. Accordingly, chronic AR has a greater impact on RV than MR.

\section{REFERENCES}

1. Vahanian A, Alfieri O, Andreotti F, et al. Guidelines on the management of valvular heart disease (version 2012): The joint task force on the management of valvular heart disease of the European Society of Cardiology (ESC) and the European Association for Cardiothoracic Surgery (EACTS). Eur Heart J 2012; 33:2451-96

2. Bonow RO, Carabello BA, Kanu C, et al. ACC/AHA 2006 guidelines for the management of patients with valvular heart disease: A report of the American College of Cardiology/American Heart Association task force on Practice Guidelines. Circulation 2006; 114:84-231

3. Le Tourneau T, Messika-Zeitoun D, Russo A, et al . Impact of left atrial volume on clinical outcome in organic mitral regurgitation, Journal of the American College of Cardiology 2010; 56(7): 570-8.

4. Nozohoor S, Hyllén S, Meurling C, et al. Prognostic value of pulmonary hypertension in patients undergoing surgery for degenerative mitral valve disease with leaflet prolapse. Journal of cardiac surgery 2012; 27(6): 668-75.

5. Hyllén S, Nozohoor S, Ingvarsson A, et al .Right ventricular performance after valve repair for chronic degenerative mitral regurgitation, The Annals of thoracic surgery $2014 ;$ 98(6): 2023-30.

6. Le TourneauT, Deswarte G, Lamblin N, et al. Right ventricular systolic function in organic mitral regurgitation: impact of biventricular impairment. Circulation 2013; 127(15): 1597-608. 
7. Khandhar S, Varadarajan P, Turk R, et al. Survival benefit of aortic valve replacement in patients with severe aortic regurgitation and pulmonary hypertension. Annals of Thoracic Surgery 2009; 88(3):752-6.

8. Weber KT, Janicki JS, Shroff S, Fishman AP. Contractile mechanics and interaction of the right and left ventricles. Am J Cardiol. 1981;47:686-695.

9. Klima U, Guerrero JL, Vlahakes GJ. Contribution of the interventricular septum to maximal right ventricular function. Eur J Cardiothorac Surg. 1998;14:250-255.

10. Nagel E, Stuber M, Hess OM. Importance of the right ventricle in valvular heart disease. Eur Heart J. 1996; 17:829-836.

11. Alexopoulos D, Lazam C, Borrico S, Fiedler L, Ambrose J. Isolated chronic mitral regurgitation with preserved systolic left ventricular function and severe pulmonary hypertension. JACC. 1989;14:319-322.

12. Borer JS, Hochreiter C, Rosen S. Right ventricular function in severe non-ischaemic mitral insufficiency. Eur Heart J. 1991;12 Suppl B:22-25.

13. Grose R, Strain J, Yipintosoi T. Right ventricular function in valvular heart disease: Relation to pulmonary artery pressure. J Am Coll Cardiol. 1983;2:225-232.

14. Costa RL, Cruz Lamas C, Manuel V et al. Impact of Pulmonary Hypertension on Surgical Mortality and 3-Year Survival After Aortic Valve Replacement. International Journal of Cardiovascular Sciences. 2016;29(1):24-30

15. Mentias A, Feng K, Alashi A, et al. Long-term outcomes in patients with aortic regurgitation and preserved left ventricular ejection fraction. JACC 2016; 68(20)
16. Gomez-Doblas JJ, Schor J , Vignola P ,et al. Left ventricular geometry and operative mortality in patients undergoing mitral valve replacement. Clin. Cardiol. 2001;24: 717-22

17. Carlsson C., Häggström J., Eriksson A., et al. Size and shape of right heart chambers in mitral valve regurgitation in small-breed dogs, Journal of veterinary internal medicine / American College of Veterinary Internal Medicine. 2009; 23(5):1007-13

18. Roscani MG, Polegato BF, Minamoto SE ,et al. Left ventricular sphericity index predicts systolic dysfunction in rats with experimental aortic regurgitation. J Appl Physiol . 2015; 116(10): 1259-62

19. Weber RT, Janicki JS , Shroff S ,et al. Contractile mechanics and interaction of the right and left ventricle. Am J Cardiol. 1981;47:686-95

20. Lopez-Candles A, Rajagopalan N , Kochar M et al. Systolic eccentricity index identifies right ventricular dysfunction in pulmonary hypertension. International journal of cardiology 2008; 129 (3) 424:6

21. Ryan T, Petrovic O, Dilon JC ,et al. An ecchocardiographic index for separation of right ventricular volume and pressure overload.J am Coll Cardiol. 1985; 5(4): 918:27

22. Morrison DA, Lancaster L ,Henry $\mathrm{R}$, et al.Right ventricular function at rest and during exercise in aortic and mitral valve disease.JACC 1985;5(1): 21-8.

23. Grose R, Strain J ,Yipintosoi T. Right ventricular function in valvular heart disease: relation to pulmonary artery pressure. J Am Cardiol. 1983;2:225-32

24. Kurr KS, Gandsman EJ, Winkler ML , et al. Hemodynamic correlates of right ventricular ejection fraction measured with gated radionuclide angiography. Am J Cardiol 1982;49:71-7 